\title{
Intento de Autoeliminación en la Niñez y Adolescencia. Presentación de un modelo de prevención del intento de autoeliminación en niños y adolescentes tempranos en un Hospital Pediátrico en Montevideo
}

\author{
Attempt of Self-elimination in Childhood and Adolescence. Presentation of a \\ model for the prevention of attempted self-elimination in children and early \\ adolescents in a Pediatric Hospital in Montevideo
}

Adriana Martínez-Schiavo (1) Laura Viola (12).

${ }^{1}$ Universidad de la República, Facultad de Medicina, Montevideo, Uruguay.

${ }^{2}$ Asociación Española, Departamento de Psiquiatría Infantil, Montevideo, Uruguay.

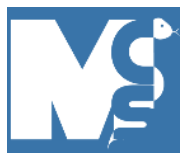

Recibido: $22 / 07 / 2021$

Revisado: 20/08/2021

Aceptado:30/08/2021

\section{Autor correspondiente}

Prof. Agda. Dra. Adriana MartínezSchiavo.

Universidad de la República,

Montevideo, Uruguay.

adrianamartinezschiavo@gmail.com

Conflictos de interés

Los autores declaran no poseer conflictos de interés.

\section{Fuente de financiación}

Los autores no recibieron apoyo financiero para la investigación, autoría y/o publicación de este artículo.

Este artículo es publicado bajo una licencia de Creative Commons

Reconocimiento 4.0 Internacional.

\section{RESUMEN}

Objetivo general: Descripción del trabajo realizado en la Policlínica Especializada en Conductas Autolesivas (PECA) de la Clínica de Psiquiatría Infantil. Presentación de un posible modelo de prevención del segundo o posteriores intentos de autoeliminación(IAE). Metodología: Estudio descriptivo, transversal de una muestra de niños y adolescentes de edades entre 6 y 15 años que consultaron por comportamientos suicidas en un periodo de 9 meses ( $n=102$ pacientes), en los años 2012-2013. Se realizaron entrevistas clínicas semiestructuradas, se aplicó la CBCL (Child Behavior Check List) y Cuestionario complementario. Resultados: El 78,8 \% de la muestra son del sexo femenino y el $75 \%$ tienen entre $12-14$ años. Primer IAE $68 \%$, segundo o más IAE $16 \%$. Escolarizados 63,6 \%. Media de PT (Problemas Totales) 66,4. El 42,3\% de los padres refieren que sus hijos son víctima de violencia. Del total de 102, 90 continúan su tratamiento. Frecuencia de reingresos $12 \%$. Un suicidio. Conclusiones: El desarrollo de un modelo de prevención del segundo o posteriores intentos de autoeliminación durante un periodo de 9 meses, evidencia la importancia de un seguimiento de proximidad. Las alteraciones emocionales y conductuales objetivadas en una población joven se favorecen por los bajos niveles de escolaridad y la violencia intrafamiliar.

Palabras clave: Intento de autoeliminación; niños y adolescentes; factores de riesgo; prevención.

\section{ABSTRACT}

General objectives: Explain the way in which the Specialized Polyclinic in Self-Injury Behaviors, works in the Child Psychiatry Clinic. Prevention of second or more suicide attempt. Methodology: Descriptive, crosssectional study of a sample of children and adolescents between the ages of 6 and 15 who consulted for suicidal behaviors in a period of 9 months (n 102 patients). Semi-structured clinical interviews were conducted and the $\mathrm{CBCL}$ (Child Behavior Check List) and supplementary questionnaire was applied. Results: Female $78,8 \%$. $12-14$ years old $75 \%$. First suicide attempt $68 \%$, second or more $16 \%$. Schooling $63,6 \%$. Mean of PT $66,4.42,3 \%$ of parents report that their children are victims of violence. Of 102 patients, 90 (88\%) continue their treatment. Frequency of readmissions $12 \%$. One suicide, $1 \%$. Conclusions: These numbers warm of a serious problem that requires great efforts to identify possible risk factors and reduce their incidence.

Keywords: Attempted suicide; children and adolescents; risk factors; prevention.

Cómo citar este artículo: Martínez-Schiavo A, Viola L. Intento de Autoeliminación en la Niñez y Adolescencia. Presentación de un modelo de prevención del intento de autoeliminación en niños y adolescentes tempranos en un Hospital Pediátrico en Montevideo. Med. clín. soc. 2021;5(3):145-151 


\section{INTRODUCCIÓN}

Cerca de 800.000 personas se suicidan cada año (1). En el Plan de Acción sobre Salud Mental 2013-2020, los Estados Miembros de la Organización Mundial de la Salud se comprometieron en alcanzar la meta de reducir globalmente, las tasas de suicidios en un $10 \%$ para 2020 (2). Sin embargo, a pesar de los esfuerzos realizados, las tasas de suicidio entre los jóvenes han aumentado tanto, que estos representan el grupo con mayor riesgo de suicidio (1).

Mundialmente el suicidio se ubica como primera o segunda causa de muerte, tanto en varones como en mujeres menores de 19 años (3). La variación en las tasas de suicidio no ha sido uniforme. En los países industrializados ha habido una leve disminución y en otros, con tasas históricamente bajas, un aumento. Diferentes hipótesis señalan tanto a los rápidos cambios económicos como a los estresores sociales (3). En Uruguay, por ejemplo, son elevadas las tasas de suicidio en comparación con las de la región (4). Estas tasas, con una media de 10/100000, se mantuvieron relativamente estables con oscilaciones no significativas, hasta la década de los 90 , en que se visualiza un paulatino aumento (5).

Las estadísticas mundiales subestiman la tasa de suicidio en niños y adolescentes (NA), que varía considerablemente no sólo por la exactitud de los registros sino, posiblemente, por diferencias de costumbres tanto culturales como legales que rodean al tema (6). Son más altas tasas en adolescentes varones que en adolescentes mujeres, con una proporción de 4:1 (7). En relación a los niños, entre los 7 y 9 años el desarrollo cognitivo y emocional facilita la comprensión del concepto de irreversibilidad de la muerte y luego de los 10 años, de universalidad (8). Acepta su propia muerte y la de sus familiares (3).

El IAE realizado en los menores de 10 años, es de mayor gravedad en sí mismo, en relación con la elección del método que atentará dramáticamente contra la integridad del cuerpo, el ahorcamiento, la defenestración, las armas de fuego $(6,9)$. Una revisión sistemática de la prevalencia en estudios comunitarios, mostró que el $20 \%$ de los niños y adolescentes manifestó haber pensado en el suicido el año anterior (10).

Dentro de los factores de riesgo, se destaca la exposición continua las situaciones de violencia y maltrato $(9,11)$ estando el abuso sexual fuertemente asociado con intentos de suicidio $y$ lesiones autoinfligidas (LAls) (12-14). A su vez, la historia parental de abuso sexual en su infancia se relaciona con un aumento del riesgo para el intento de suicidio del hijo (15). Los factores de estrés familiar desempeñan un papel significativo, repercutiendo en las tempranas conductas de apego, en las dificultades de los cuidados y en la comunicación verbal (16-18).

El vínculo entre el suicidio y los trastornos mentales, no siempre evidente (4), parecería responder a la acumulación de factores de riesgo que repercute tanto en los problemas internalizados como externalizados (7) y las situaciones individuales estresantes marcadas por la impulsividad (19). En el suicidio infantil, el Trastorno Psiquiátrico presente alrededor de un $80 \%$, se relaciona los trastornos del estado de ánimo, de ansiedad, del comportamiento y del consumo de sustancias $(3,9)$. La desregulación emocional, propia de las patologías graves del humor y del comportamiento de presentación temprana, se asocian a los IAE y a las LAls $(19,20)$.

El fracaso escolar y/o el abandono han sido señalados como factores de riesgo para el IAE por diversos investigadores $(15,21,22)$. En el análisis de los datos de adolescentes que realizaron IAE y/o suicido, se destacan, en relación con los que no lo realizaron, mayores dificultades escolares $y / o$ abandono de la educación formal (23).

Las experiencias relacionadas con conflictos, desastres, violencia, abusos, pérdidas y sensación de aislamiento están estrechamente ligadas a conductas suicidas. Las tasas de suicidio son elevadas entre aquellos grupos vulnerables por ser objeto de discriminación y exclusión (1).

Los factores de protección frente a las adversidades se relacionan fuertemente con la presencia de vínculos cercanos, tanto en la experiencia propia desarrollada como la que aporta la familia, la escuela o el entorno $(8,13)$. La OMS plantea que la prevención de los suicidios es posible si se incorpora una estrategia integral multisectorial como un componente central en los servicios de salud (2). Existen dos metaanálisis que arrojan respuestas que toman en cuenta las dificultades en la prevención. Large y colaboradores (23) revisaron estudios longitudinales de cohortes de pacientes psiquiátricos o personas con intentos de suicidio estratificados en grupos de alto y bajo riesgo para el suicidio. Después de un seguimiento medio de seis años, el 5,5\% de los participantes en el grupo de alto riesgo frente al $1 \%$ de los del grupo de bajo riesgo se habían suicidado. La precisión de la predicción fue baja y la combinación de múltiples factores de riesgo para el suicidio no excedió sustancialmente el poder predictivo de los factores de riesgo individuales.

Chan et al (24) examinaron estudios prospectivos sobre factores de riesgo y escalas de evaluación para predecir 
el suicidio después de una autolesión. De nuevo, la predicción fue baja y los factores de riesgo estadísticamente significativos (autolesiones repetidas, intención suicida, género masculino, problemas de salud física) son comunes en poblaciones clínicas y no específicos para tendencias suicidas. No hay aún métodos estadísticos fiables que permitan una predicción precisa del suicidio. Los autores concluyen que la categorización de alto o bajo riesgo de suicidio, basada en escalas, puede no ser precisa y no ser recomendable $(7,24)$.

En los servicios de Psiquiatría Pediátrica, uno de los frecuentes motivos de interconsulta es el IAE (25). La Clínica de Psiquiatría Infantil, desde hace años ha centrado el esfuerzo en la prevención del segundo o posteriores IAE, entendiendo a éstos como el factor predictor de mayor riesgo para el suicidio (24). Se promovió, para tal fin, la creación de una policlínica especializada en Conductas Autolesivas (PECA). El presente artículo describe la labor realizada en la Policlínica Especializada en Conductas Autolesivas y su utilización como insumo de un posible modelo de prevención del suicidio, evitando el segundo o posteriores Intentos de autoeliminación.

\section{METOdOLOGÍA}

Estudio descriptivo y transversal de todos los menores de 15 años que consultaron en Puerta de Emergencia del CHPR y fueron internados en Pediatría por IAE, LAls y equivalentes suicidas, en un período de 9 meses de octubre 2012 a julio 2013 (n:102). En las primeras 24 horas de la internación, si las condiciones orgánicas lo permiten, se realiza una entrevista clínica semiestructurada, y se entrega la CBCL-P y YSR-11 a 18 años. Se ha elegido para el control y seguimiento de las alteraciones emocionales y conductuales, al listado de la Child Behavior Check List -CBCL-, que integra los instrumentos ASEBA creados por Achenbach (26), por su confiabilidad y validez para evaluar poblaciones tanto clínicas como no referidas. Su viabilidad o fortaleza transcultural ha quedado demostrada $y$ documentada a través de estudios epidemiológicos llevados a cabo en múltiples países (27) y en Uruguay (28).

Esta escala, que consta de 112 ítems, es completada por los padres (CBCL-P) o referentes y por los niños mayores de 11 años CBCL-Y. Es de fácil comprensión y aplicación. Tanto en las escalas de competencia como en los síndromes empíricos (29) y los trastornos orientados por el DSM, el puntaje bruto obtenido por un sujeto se clasifica en nivel "clínico o patología", "riesgo" o "normalidad", en función de percentiles y puntuaciones $T$ derivadas de la muestra normativa del estudio llevado a cabo al construir y baremar el instrumento. La sumatoria de 112 ítems da un número, Problemas Totales (PT), índice de psicopatología, que da cuenta de la gravedad. La Media aritmética (X்TP) es una medida muy útil para comparar entre poblaciones de una misma muestra o de diferentes procedencias y para evaluar la evolución del paciente.

La CBCL utiliza dos tipos de categorías diagnósticas, la primera propia, -síndromes empíricos- de la escala que agrupa a los ítems en 8 dimensiones y la segunda que se basa en el DSM (30), agrupada en 6 trastornos, siendo esta última la que se utilizará (31). La escala de CBCL Padres como la de Youth Self Report, que es la que llenan los adolescentes mayores de 11 años, tienen las mismas variables. En la de Padres es la percepción de padres o adultos responsables, de las alteraciones que presenta su hijo y en la CBCL-Y es la percepción que tiene el adolescente de sus propios problemas emocionales. La concordancia entre ambas escalas es alta (32). Ambos instrumentos permiten capturar el Índice de Suicidabilidad (3). Se entiende por suicidabilidad las cogniciones y actividades en la búsqueda de la propia muerte incluyendo pensamientos y acciones.

La variable de suicidabilidad se construye con la sumatoria simple de las variables Q18 que indaga sobre las acciones: "¿Se hace daño a si mismo deliberadamente o ha intentado suicidarse?" y la Q91, “¿Habla de querer matarse?" que da cuenta de las verbalizaciones. Se aplica un Cuestionario Complementario, diseñado por el equipo de la PECA, de las variables socio-demográficas y aquellas que han demostrado una fuerte asociación con el IAE $(14,17$, 21).

\section{RESULTADOS}

Se realizó la atención de los 102 niños que concurrieron a la PECA en el período señalado. Para esta investigación se excluyeron los niños sin referentes con información válida, los niños cuyos padres se negaron a completar los cuestionarios, y los que no aceptaron la internación. Se excluyeron 22 pacientes. La muestra resultante es de $n=80$ pacientes. Todos provienen de un medio socio-económico bajo y/o marginal. De la muestra de 80 niñas/os y adolescentes, $n=63$ (78,8 \%) pertenecía al sexo femenino y $n=17(22,2 \%)$ al sexo masculino. El rango de edad es de 6 a 15 años, con una moda de 14 años y una media de 12 años y 4 meses.

En referencia a la edad, el $75 \%$ de los pacientes tenían entre 12 y 14 años. El $12,5 \%$ de la muestra se encuentra entre los 6 y 8 años, siendo entre los 9 y 11 años el $10 \%$ y con 15 años el 2,5\%, posiblemente en relación con el límite de edad para el ingreso o la 
consulta en el Centro Hospitalario Pereira Rossell. En referencia al motivo de interconsulta, el $65 \%$ es un primer IAE. En el $16 \%$ fue el segundo o más IAE, gesto o conductas de riesgo $5 \%$ y amenazas $5 \%$, LAls 3,8\%. El $55 \%$ de los pacientes realizaron el Intento de AE con psicofármacos, de uso de algún miembro de la familia. En un $10 \%$ de uso personal.

En relación a la escolarización: un 7,5 \% de la muestra no está escolarizada. El $35 \%$ de los varones tienen retraso escolar (repitieron un año o más) y lo mismo sucede con el $52 \%$ de las mujeres. La media de Problemas Totales (XंTP) es de 67,3 lo que da cuenta de la gravedad de la muestra. De acuerdo a CBCLP no hay una diferencia estadísticamente significativa por sexo, entre varones XंTP 67,3 y mujeres XंTP 63,9 ( $p<0,05)$.

En los adolescentes la percepción de problemas entre las mujeres es mayor que entre los varones con una diferencia significativa medido por la media de XंTP. EI $79,7 \%$ de los padres consignan la presencia de patología en sus hijos. Los adolescentes se reconocen en un $48,3 \%$ con problemas emocionales o conductuales. Dicho de otro modo, un $51,7 \%$ de los adolescentes internados por un IAE, consideran que no tienen problemas. El trastorno del humor es predominante tanto en la CBCL-P (74 \%) como en la YSR (53\%).

El Trastorno por Estrés Post Trauma (TEPT) o Disregulation Short Scale (DSS) es una variable muy sensible que captura los pacientes desregulados con trastornos graves del humor y del comportamiento (20). Los padres perciben que un $66 \%$ tiene una desregulación, mientras que la percepción del niño o adolescente es de la mitad $37 \%$.

Otra de las variables usadas es el índice de suicidabilidad: Q18 que indaga sobre las acciones: “¿Se hace daño a si mismo deliberadamente o ha intentado suicidarse?” y la Q91, “¿Habla de querer matarse?” que da cuenta de las verbalizaciones. El 42,3\% de los padres refieren que sus hijos fueron víctimas de violencia, considerando la Violencia Intra -familiar y ser Testigos u Objeto de la misma. El Fallecimiento de la madre: $3,8 \%$ y del padre: $11,3 \%$ han sido especialmente consignados por los adultos como factor de riesgo o desencadenante. La evolución y seguimiento se puede ver en la Tabla 1.

TABLA 1. EVOLUCIÓN Y SEGUIMIENTO DE LOS PACIENTES ( $\mathrm{N}=102)$

\begin{tabular}{lcc}
\hline & Cantidad de niños - adolescentes \\
\hline & Luego del ingreso \\
\hline Derivados a clínicas de Agudo & 5 & $4,9 \%$ \\
Fugas del hospital & 7 & $6,8 \%$ \\
Derivados a policlínicas zonales & 5 & $4,9 \%$ \\
\hline \multicolumn{3}{c}{ Luego del alta } \\
\hline Atención en PECA & 79 & $77,4 \%$ \\
Abandono del tratamiento & 6 & $5,8 \%$ \\
Mantienen contacto telefónico & 48 & $47 \%$ \\
Reingreso por IAE & 12 & $11,7 \%$ \\
Suicidio & 1 & $0,9 \%$ \\
\hline
\end{tabular}

\section{DISCUSIÓN}

La tercera parte de la muestra pertenece al sexo masculino, con una relación de 3 a 1 acorde con los datos internacionales. El $50 \%$ de los varones que realizan un IAE tienen 10 años o menos, mientras que las mujeres es el $8 \%$. Se destaca el porcentaje del segundo o posterior IAE, $16 \%$ de la muestra, por el riesgo que significa como predictor para el suicidio (24). Estos datos son impactantes considerando la edad referida (menores de 10 años). Es ésta, una población de niños y adolescentes, en general de bajo nivel socioeconómico y educativo, donde el seguimiento y control del cumplimiento del tratamiento psiquiátrico psicológico y social se debe facilitar. El medio socioeconómico bajo o marginal es un factor de riesgo en la salud mental (33), al igual que la violencia, ser testigo u objeto de la misma (11). La disfunción familiar que esto origina al igual que la muerte de los padres es un factor desestabilizador de los trastornos tanto internalizados o externalizados subyacentes (7).

En esta muestra hay un importante retraso escolar, las dificultades académicas y el abandono escolar están señalados como fuertes factores de riesgo para las conductas lesivas $(2,15,19,21,22,25)$. Si bien el $92,5 \%$ se encuentran escolarizados, lo cual se podría considerar como un factor protector, en cuanto a su nivel de instrucción, el $35 \%$ de los varones tienen retraso escolar (o sea repitieron un año) y lo mismo sucede con el $52 \%$ de las mujeres. Abandonar la escuela debe ser entendido en el marco de un 
complejo sistema de pérdidas y frustraciones en el niño y no sólo como expresión de una dificultad del aprendizaje (6). En los datos empíricos de esta investigación las mujeres se perciben con más alteraciones emocionales. La causalidad entre esta percepción de las mujeres y las alteraciones académicas debería profundizarse en próximas investigaciones. Posiblemente el tamaño de la muestra en mujeres, más del triple que varones, explique esta asociación.

La Media de PT (Problemas Totales) para población total de niños en Uruguay es de 33,9 y una Media de PT en medio socioeconómico bajo de 39,8, como fue señalado en estudios anteriores de la Clínica de Psiquiatría Pediátrica $(28,34)$. Es interesante subrayar la percepción tanto de los padres como de los adolescentes de sus problemas emocionales y conductuales. Como fue mostrado, más de la mitad de los adolescentes $(51,7 \%)$, consideran que no tienen problemas emocionales o conductuales que ameritaran su atención. Es un punto de gran riesgo, ya que son niños y adolescentes que se encuentran ingresados por alguna conducta donde pusieron en riesgo su vida y no pueden comprender el riesgo incluso luego de la internación.

De acuerdo a las escalas utilizadas, el predominio de la percepción, tanto por adolescentes como por los padres de los trastornos del humor, $53 \%$ y $74 \%$ respectivamente, evidencia una mayor posibilidad de los padres de ver el sufrimiento de sus hijos en comparación de la percepción del adolescente de valorar su propio sufrimiento. Situación que se repite en todas las demás áreas de diagnóstico, donde se ve que el Trastorno de ansiedad, el de conducta, el oposicionista desafiante y el déficit atencional con hiperactividad en la CBCL-P se encuentran en el entorno del $50 \%$ y en la CBCL-Y en el $20 \%$. Los padres consideran que el $74 \%$ de los adolescentes presentan Trastorno del Humor. En contraposición con los adolescentes un $52,5 \%$ reconoce en ellos el Trastorno del Estado de Ánimo.

En relación al índice de Suicidabilidad, en el análisis de las respuestas brindadas por los padres se observa que el $28,8 \%$ responden en forma negativa (no es cierto) a la pregunta "¿ha intentado hacerse daño?", siendo que el niño/adolescente está ingresado por ese motivo. Mientras que el niño/adolescente frente a la misma pregunta responden que no es cierto el $14,5 \%$, por lo tanto, muestran una relativa mejor percepción de la situación.

Mientras en lo que se refiere al ítem que indaga verbalizaciones en ambas escalas, alrededor del $50 \%$, no verbalizan un deseo de quitarse la vida, lo que lleva a reflexionar sobre la tendencia del pasaje al acto del adolescente (7) y la necesidad de estar atentos a las señales de sufrimiento. Es entonces, imperativo insistir sobre la negación del acto, del sufrimiento y del riesgo, tanto con el paciente como con su familia. Al momento de la evaluación inicial, este aspecto de la negación de lo que sucede, debe ser considerado como un objetivo principal en el proceso diagnóstico terapéutico que se inicia en el momento del ingreso.

No siempre son evidentes las alteraciones emocionales. La conducta suicida también puede ocurrir en niños y adolescentes sin un trastorno psiquiátrico (4). Es de señalar que no deben desestimarse, en estas situaciones de vulnerabilidad y frustración, los recursos emocionales propios del niño, la resiliencia, las experiencias positivas tempranas que hayan permitido establecer un apego seguro que funcione como protector frente al sufrimiento.

Estrategias de PECA. Se tomó en cuenta las recomendaciones internacionales de incorporar un abordaje integral multisectorial de prevención del suicidio como un componente central en la atención (2). En esta población referida al área de salud mental, tanto desde los servicios de salud y de los educativos, se deberá estar atento a la presencia de posibles conductas autolesivas. En la práctica clínica, es indispensable una evaluación clínica exhaustiva del paciente que cubra los riesgos y las necesidades individuales $(7,24)$. En este contexto, se le da un lugar relevante a la prevención, es importante considerar lo que se podría denominar una historia de "tapar" el suicido. Los cuales no se evitan callando. El adolescente necesita ser escuchado en su sufrimiento.

La tendencia hacia la vida presente en el posible suicida se puede palpar en las demandas de escucha, hay un otro que debe escuchar. De lo contrario corre el adolescente el riesgo de pasar al acto. Se puede considerar el IAE como un llamado para hacerse oír, donde el dolor y el sufrimiento psíquico del niñoadolescente, se vincula al actuar (35). El estar en el camino de la prevención exige aprender a escucharlas. La estrategia del control del seguimiento se compone de dos pilares, por un lado, mantener la atención luego del alta con el psiquiatra pediátrico que lo atendió durante su internación. En segundo lugar, el control telefónico (cada 3 meses) que tiene por finalidad tanto mantener el vínculo con el paciente evaluando la presencia de desencadenantes que configuren un riesgo como el recordatorio de las citas pautadas. Las llamadas telefónicas han demostrado su utilidad como complemento en el control de los adolescentes con conductas suicidas (36). 
La preocupación del técnico que trabaja con adolescentes que son o pueden ser suicidas, es cuándo preocuparse y cuándo es urgente la ayuda. Cuando un niño-adolescente dice "quiero morir" no hay que desestimar ni magnificar esta afirmación. El adolescente busca a un otro que se haga presente. Se trabaja con la certeza que, en muchas situaciones, independientemente de las respuestas que reciba, hay adolescentes que están decididos a morir y los esfuerzos del clínico, serán en vano.

\section{CONTRIBUCIÓN DE LOS AUTORES}

Todos los autores participaron en la redacción del borrador, revisión crítica del manuscrito y aprobación final del mismo.

\section{REFERENCIAS}

1. OMS. Suicidio. https://wwwwhoint/es/news-room/factsheets/detail/suicid [Internet]. 2019.

2. OMS. Prevención del suicido. Un imperativo Global. https://wwwwhoint/mental health/suicideprevention/es/ [Internet]. 2014.

3. Jans $T$, Timo $T$. Trastornos del estado de ánimo. Suicidio y conducta autolesiva. In: Irrazabal M, Martin A, editors. Manual de Salud Mental Infantil y Adolescente 2018 ed: IACAPAP; 2018. p. 1-40.

4. Canetti A. La conducta suicida desde la perspectiva psiquiátrica. Una revisión de la situación en Uruguay. In: CSIC., editor. 70 años de Suicido en Uruguay: 7 disciplinas, 7 entrevistas, 7 encuentros. Uruguay2017. p. 107-34.

5. Larrobla C. Adolescencia y suicidio: su abordaje como fenómeno complejo desde el Sector Salud. In: ImprentaRojo, editor. 70 años de Suicidio en Uruguay: 7 disciplinas, 7 entrevistas, 7 encuentros. Uruguay: CSIC Universidad de la república; 2017. p. 31-46.

6. Hawton K. Suicidal Behaviour and self-harm. In: Sons JW, editor. Rutter"s Child and Adolescent Psychiatry. Sixth Edition ed. EUA: John Wiley \& Sons, Ltd; 2015. p. 893-912.

7. Shin YM, Chung YK, Lim KY, Lee YM, Oh EY, Cho SM. Childhood predictors of deliberate self-harm behavior and suicide ideation in korean adolescents: a prospective population-based follow-up study. J Korean Med Sci. 2009;24(2):215-22.

https://doi.org/10.3346/jkms.2009.24.2.215

8. Bering JM, Blasi CH, Bjorklund DF. The development of afterlife beliefs in religiously and secularly schooled children. British Journal of Developmental Psychology. 2005;23(4):587-607. https://doi.org/10.1348/026151005×36498

9. Rodriguez-Almada H, García-Maggi I, Ciriacos C. Resultados de la aplicación de la autopsia psicológica al estudio del suicidio de niños y adolescentes en Uruguay. Rev Med Uruguay 2005;21:141-50. URL.

10. Joe $S$, Romer D, Jamieson PE. Suicide acceptability is related to suicide planning in US adolescents and young adults. Suicide and Life-Threatening Behavior. 2007;37(2):165-78.

https://doi.org/10.1521/suli.2007.37.2.165

11. Viola L. Repercusión de la violencia doméstica en la salud mental infantil. Valoración del daño psíquico. Rev Psiquiatr Urug 2010;74(1):73-83. URL.

12. Beautrais AL. Child and Young Adolescent Suicide in New
Zealand. Australian \& New Zealand Journal of Psychiatry. 2001;35(5):647-53.

https://doi.org/10.1080\%2F0004867010060514

13. Taliaferro LA, Muehlenkamp JJ, Borowsky IW, McMorris BJ, Kugler KC. Factors Distinguishing Youth Who Report SelfInjurious Behavior: A Population-Based Sample. Academic Pediatrics. https://doi.org/10.1016/j.acap.2012.01.008

14. Isohookana R, Riala K, Hakko H, Räsänen P. Adverse childhood experiences and suicidal behavior of adolescent psychiatric inpatients. European child \& adolescent psychiatry.

2013;22(1):13-22.

https://doi.org/10.1007/s00787-012-0311-8

15. Donald M, Dower J, Correa-Velez I, Jones M. Risk and Protective Factors for Medically Serious Suicide Attempts: A Comparison of Hospital-Based with Population-Based Samples of Young Adults. Australian \& New Zealand Journal of Psychiatry. 2006;40(1):87-96. https://doi.org/10.1080/j.1440-1614.2006.01747.x

16. Fergusson DM, Lynskey MT. Childhood circumstances, adolescent adjustment, and suicide attempts in a New Zealand birth cohort. Journal of the American Academy of Child \& Adolescent Psychiatry. 1995;34(5):612-22. https://doi.org/10.1097/00004583-199505000-00013

17. Fergusson $D$, Woodward L. Risk factors and life processes associated with the onset of suicidal behaviour during adolescence and early adulthood. Psychological medicine. 2000;30(1):23-39.

https://doi.org/10.1017/s003329179900135x

18. Ackard DM, Neumark-Sztainer D, Story M, Perry C. Parent-child connectedness and behavioral and emotional health among adolescents. American journal of preventive medicine.

2006;30(1):59-66.

https://doi.org/10.1016/j.amepre.2005.09.013

19. Zahid S, Bodicherla K, Eskander N, al e. AttentionDeficit/Hyperactivity Disorder and Suicidal Risk in Major Depression: Analysis of 141,530 Adolescent Hospitalizations. Cureus 12(5. 2020;12(5). https://dx.doi.org/10.7759\%2Fcureus.7949

20. Ayer L, Althoff R. Child Behavior Checklist Juvenile Bipolar Disorder (CBCL-JBD) and CBCL Posttraumatic Stress Problems (CBCL-PTSP) scales are measures of a single dysregulatory syndrome. Journal of child psychology and psychiatry, and allied disciplines. 2009 50(10)(1):291-300. https://doi.org/10.1111/i.1469-7610.2009.02089.x

21. Tang $\mathrm{T}-\mathrm{C}$, Ko C-H, Yen J-Y, Lin H-C, Liu S-C, Huang C-F, et al. Suicide and Its Association with Individual, Family, Peer, and School Factors in an Adolescent Population in Southern Taiwan. Suicide and Life-Threatening Behavior. 2009;39(1):91-102. https://doi.org/10.1521/suli.2009.39.1.91

22. Daniel SS, Walsh AK, Goldston DB, Arnold EM, Reboussin BA, Wood FB. Suicidality, School Dropout, and Reading Problems Among Adolescents. Journal of Learning Disabilities. 2006;39(6):507-14. https://doi.org/10.1177/00222194060390060301

23. Large M, Kaneson M, Myles N, Myles H, Gunaratne P, Ryan C. Meta-Analysis of Longitudinal Cohort Studies of Suicide Risk Assessment among Psychiatric Patients: Heterogeneity in Results and Lack of Improvement over Time. PLOS ONE 2016;11(6). https://doi.org/10.1371/journal.pone.0156322

24. Chan MK, Bhatti H, Meader N, Stockton S, Evans J, O'Connor RC, et al. Predicting suicide following self-harm: systematic review of risk factors and risk scales. $\mathrm{Br} \mathrm{J}$ Psychiatry

2016;209(4):277-83. 
https://doi.org/10.1192/bjp.bp.115.170050

25. Martínez-Schiavo A, Goyeneche N, Kehyaian V, Lungo G, Viola L. El intento de autoeliminación en el Centro Hospitalario Pereira Rossell. Revista de APPIA. 2009;18(2):92-8.

26. Achenbach TM, Rescorla LA. Manual for the ASEBA School-Age Forms \& Profiles. Burlington: University of Vermont, Research Center for Children, Youth, \& Families; 2001.

27. Rescorla L, Ivanova MY, Achenbach TM, Begovac I, Chahed $M$, Drugli $M B$, et al. International epidemiology of child and adolescent psychopathology II: integration and applications of dimensional findings from 44 societies. Journal of the American Academy of Child \& Adolescent Psychiatry. 2012;51(12):1273-83.

e8. https://doi.org/10.1016/j.jaac.2012.09.012

28. Viola L, Garrido G, Rescorla L. Testing multicultural robustness of the Child Behavior Checklist in a national epidemiological sample in Uruguay. Journal of abnormal child psychology. 2011;39(6):897-908. https://doi.org/10.1007/s10802-011-9500-z

29. Verhulst $F$, Achenbach TM. Empirically based assessment and taxonomy of psychopathology: cross-cultural applications. A review. European Child and Adolescent Psychiatry 1995:61-76. https://doi.org/10.1007/bf01977734

30. American Psychiatry Association. Diagnostic and Statistical Manual of Mental Disorders, DSM-5. 2013.

31. Ivanova MY, Dobrean A, Dopfner M, Erol N, Fombonne $E$, Fonseca AC, et al. Testing the 8-syndrome structure of the child behavior checklist in 30 societies. Journal of clinical child and adolescent psychology : the official journal for the Society of Clinical Child and Adolescent Psychology, American Psychological Association, Division 53. 2007;36(3):405-17. https://doi.org/10.1080/15374410701444363

32. Petot D, Rescorla L, Petot JM. Agreement between parent- and self-reports of Algerian adolescents' behavioral and emotional problems. Journal of adolescence. 2010;34(5):977-986.

https://doi.org/10.1016/i.adolescence.2010.11.011

33. Viola L, Garrido G, Curone G, Roca G, Varela A. Evaluación de las características emocionales y conductuales en niños provenientes de un medio socio-económico crítico. International ASEBA Conference on Empirically based Mental Health Knowledge 21-24 june, 2009; Burlington Vermont, USA.2009.

34. Viola L, Garrido G, Varela A. Estudio Epidemiológico sobre la Salud Mental de los niños uruguayos. Montevideo Uruguay: Imprenta GEGA S.R.L; 2007.

35. Read L, Garfinkel B. Adolescent suicidal behavior: understanding the breadth of the problem in Child and Adolescents Child and Adolescents Psychiatric Clinics of North America. 1992;1(1):197-228. https://psycnet.apa.org/record/2000-12085-011

36. Biernesser C, Wolfe KL, Foxwell AA, Craddock Lee SJ, Rial KV, Patel S, et al. Developing a Brief Suicide Prevention Intervention and Mobile Phone Application: A Qualitative Report. Journal of Technology in Human Services. 2015;33(4):345-57.

https://dx.doi.org/10.1080\%2F15228835.2015.1106384 